Publ. RIMS, Kyoto Univ.

12 (1976), 383-390

\title{
Scattering Theory for Wave Equations with Dissipative Terms
}

\author{
$\mathrm{By}$
}

Kiyoshi MocHIZUKI*

\section{§1. Introduction}

We shall consider wave equations of the form

$$
w_{1 l}(x, t)+b(x, t) w_{t}(x, t)-\Delta w(x, t)=0,
$$

where $x \in \boldsymbol{R}^{n}(n \neq 2), t \geq 0, w_{t}=\partial w / \partial t, w_{t t}=\partial^{2} w / \partial t^{2}$ and $\Delta$ is the $n$ dimensional Laplacian. $b(x, t)$ is a non-negative function and is assumed to satisfy the following conditions:

(A1) There exist constants $C_{1}>0$ and $\delta>0$ such that

$$
0 \leq b(x, t) \leq C_{1}(1+|x|)^{-1-\hat{o}} \text { for any } x \in \mathbb{R}^{n}, t \geq 0 .
$$

(A2) $b_{t}(x, t)$ is bounded continuous in $x \in \boldsymbol{R}^{n}$ and $t \geq 0$.

In the following we assume that $\delta \leq 1$ without any loss of genelarity. Since $b(x, t) \geq 0, b(x, t) w_{t}(x, t)$ represents the resistance of viscous type. Our aim of this note is to show that the solutions of $(1 \cdot 1)$ are asymptotically equal for $t \rightarrow \infty$ to those of the free wave equation

$$
r w_{t t}^{0}(x, t)-J w^{0}(x, t)=0 .
$$

More precisely, we shall show the existence of the $M \phi 1$ ler wave operators.

We restrict ourselves to solutions with finite energy. For pars $f=\left\{f_{1}, f_{2}\right\}$ of functions in $\boldsymbol{R}^{n}$ the energy is defined by

$$
\|f\|_{E}^{2}=\int_{R^{n}}\left(\left|D f_{1}\right|^{2}+\left|f_{2}\right|^{2}\right) d x,
$$

where $D f_{1}=\left(D_{1} f_{1}, \cdots, D_{n} f_{1}\right) \quad\left(D_{j}=\partial / \partial x_{j}\right) \quad$ and $\quad\left|D f_{1}\right|^{2}=\sum_{j=1}^{n}\left|D_{j} f_{1}\right|^{2}$. The Hilbert space $\mathcal{H}$ is defined as the completion in the energy norm of

Communicated by S. Matsuura, February 5, 1976.

* Department of Mathematics, Nagoya Institute of Technology, Gokiso, Showa-ku, Nagoya 466, Japan. 
smooth data with bounded support in $\mathbb{R}^{n}$. Put $u=\left\{w, w_{t}\right\}$. Then (1.1) can be expressed in the matrix notation as

$$
u_{t}=\Lambda(t) u=\Lambda_{0} u-V(t) u,
$$

where

$$
\Lambda_{0}=\left(\begin{array}{ll}
0 & 1 \\
\Delta & 0
\end{array}\right) \text { and } V(t)=\left(\begin{array}{cc}
0 & 0 \\
0 & b(x, t)
\end{array}\right) \text {. }
$$

Put $u^{0}=\left\{w^{0}, w_{t}^{0}\right\}$. Then $(1 \cdot 2)$ is expressed as

$$
u_{t}^{0}=\Lambda_{0} u^{0} .
$$

$\Lambda_{0}$ determines a skew-selfadjoint operator in $\mathscr{H}$ with domain

$$
\mathscr{D}\left(\Lambda_{0}\right)=\left\{f \in \mathcal{H} ; \Delta f_{1}, D_{j} f_{2} \in L^{2}\left(\mathbb{R}^{n}\right) \quad(j=1, \cdots, n)\right\},
$$

where all the derivatives are considered in the distribution sense. Thus, $\Lambda_{0}$ generates a one-parameter group $\left\{U_{0}(t)=e^{\Lambda_{0} t} ; t \in \mathbb{R}\right\}$ of unitary operators. Under the above conditions on $b(x, t), \Lambda(t)$ determines for each $t \geq 0$ a closed operator in $\mathcal{H}$ with domain $\mathscr{D}(\Lambda(t))=\mathscr{D}\left(\Lambda_{0}\right)$. Moreover, positive numbers belong to the resolvent set of each $\Lambda(t)$ and $\Lambda(t)(\Lambda(0)-I)^{-1}$, where $I$ is the identity in $\mathcal{H}$, is continuously differentiable in $t$ in operator norm. Thus applying results of Kato [2], we see that there exists a unique family $\{U(t, s) ; t \geq s \geq 0\}$ of contraction evolution operators which is defined as mapping solution data of (1.4) at time $s$ into those at time $t$.

Now the main results can be stated as follows:

\section{Theorem 1. (a) The wave operator}

$$
Z=\text { strong } \lim _{t \rightarrow \infty} U_{0}(-t) U(t, 0)
$$

exists. (b) $Z$ is a not identically vanishing contraction operator in $\mathcal{H}$. (c) If we denote by $Z^{*}$ the adjoint of $Z$, then

$$
Z^{*}=\text { strong } \lim _{t \rightarrow \infty} U(t, 0) * U_{0}(t) .
$$

We also consider the special case where $b(x, t)$ is independent of $t$. Then the operator $\Lambda=\Lambda_{0}-V$, where $V=\left(\begin{array}{cc}0 & 0 \\ 0 & b(x)\end{array}\right)$, generates a semi-group $\{U(t) ; t \geq 0\}$ of contraction operators. 
In this case we have the following

Theorem 2. (a) The wave operators

$$
\begin{aligned}
& W=\text { strong } \lim _{t \rightarrow \infty} U(t) U_{0}(-t), \\
& Z=\text { strong } \lim _{t \rightarrow \infty} U_{0}(-t) U(t)
\end{aligned}
$$

exist. (b) They both are not identically vanishing contraction operators in $\mathcal{H}$. (c) $U_{0}(t)$ and $U(t)$ are intertwined by both $W$ and $Z$, i.e.,

$$
W U_{0}(t)=U(t) W, Z U(t)=U_{0}(t) Z \text { for any } t \geq 0 \text {. }
$$

(d) The scattering operator, defined by $S=Z W$, commutes with $U_{0}(t)$ :

$$
S U_{0}(t)=U_{0}(t) S \text { for any } t \in \boldsymbol{R} \text {. }
$$

The proof of these theorems will be based on the "smooth perturbation theory" developed by Kato [3].

The above theorems generalize some results already announced in Mochizuki [7], where the main concern was in the local energy decay for wave equations with non-linear dissipative terms. The scattering theory has been developed by Lax-Phillips [4] for wave equation: $w_{t t}=\Delta w$ in an exterior domain of $\boldsymbol{R}^{n}(n \geq 2)$ with lossy boundary conditions: $w_{n}$ $+\alpha(x) w_{t}=0, \alpha(x) \geq 0$. Some related problems has been studied in [1] and $[5]$.

\section{$\S 2$. Preliminaries}

First we shall show an inequality for $L^{2}$-solutions of the Helmholtz equation

$$
-\Delta u-\kappa^{2} u=f(x) \text { in } \mathbb{R}^{n},
$$

where $\kappa$ is a complex number such that $\operatorname{Im} \kappa \neq 0$ and $f(x)$ is a function such that $(1+|x|)^{(1+\delta) / 2} f(x) \in L^{2}\left(\boldsymbol{R}^{n}\right)$.

Lemma 2. 1. Let $\operatorname{Im} \kappa \gtrless 0$. Then we have for any $\rho>0$ 


$$
\begin{aligned}
& \frac{1}{2} \int_{S_{\rho}}\left(\left|\frac{\partial u}{\partial r}+\frac{n-1}{2 r} u\right|^{2}+|\kappa|^{2}|u|^{2}\right) d S \\
& \quad+|\operatorname{Im} \kappa| \int_{K_{\rho}}\left(|D u|^{2}+\frac{n-1}{2 r}|u|^{2}+|\kappa|^{2}|u|^{2}\right) d x \\
& \quad=\frac{1}{2} \int_{S_{\rho}}\left|\theta_{ \pm}\right|^{2} d S \mp \int_{K_{\rho}} \operatorname{Re}[f \bar{i} \bar{u}] d x,
\end{aligned}
$$

where $r=|x|, S_{\rho}=\{x ;|x|=\rho\}, K_{\rho}=\{x ;|x|<\rho\}$ and

$$
\theta_{ \pm}=\frac{\partial u}{\partial r}+\frac{n-1}{2 r} u \mp i \kappa u \text {. }
$$

Proof. Note the identity

$$
-\operatorname{Re}\left[\frac{\partial u}{\partial r} \overline{i \kappa u}\right]=-\operatorname{Im} \kappa \frac{n-1}{2 r}|u|^{2} \pm \frac{1}{2}\left|\theta_{ \pm}\right|^{2} \mp \frac{1}{2}\left(\left|\frac{\partial u}{\partial r}+\frac{n-1}{2 r} u\right|^{2}+|\kappa|^{2}|u|^{2}\right) .
$$

Then $(2 \cdot 2)$ follows from the integration by parts of $(2 \cdot 1)$ multiplied by $\overline{i \kappa u}$.

Lemma 2.2. Let $\operatorname{Im} \kappa \gtrless 0$. Then re have

$$
\begin{aligned}
& |\operatorname{Im} \kappa| \int_{\boldsymbol{R}^{n}} r^{\delta}\left\{\left|\zeta_{ \pm}\right|^{2}+\frac{(n-1)(n-3)}{4 r^{2}}|u|^{2}\right\} d x \\
& \quad+\int_{\mathbb{R}^{n}} r^{-1+\delta}\left\{\left(1-\frac{\delta}{2}\right)\left(\left|\zeta_{ \pm}\right|^{2}-\left|\theta_{ \pm}\right|^{2}\right)+\frac{\delta}{2}\left|\theta_{ \pm}\right|^{2}\right\} d x \\
& +\frac{(n-1)(n-3)(2-\delta)}{8} \int_{\boldsymbol{R}^{n}} r^{-3+\delta}|u|^{2} d x=\int_{\mathbb{R}^{n}} r^{\delta} \operatorname{Re}\left[f \overline{\theta_{ \pm}}\right] d x,
\end{aligned}
$$

where

$$
\zeta_{ \pm}=D u+\frac{n-1}{2 r} \frac{x}{r} u \mp i \kappa \frac{x}{r} u
$$

Proof (cf., Mochizuki [6]). Put $v=e^{\mp i \kappa r} r^{(n-1) / 2} u$. Then

$$
-\Delta v+\left(\frac{n-1}{2} \mp 2 i \kappa\right) \frac{\partial v}{\partial r}+\frac{(n-1)(n-3)}{4 r^{2}} v=e^{\mp i \kappa r} r^{(n-1) / 2} f .
$$

Multiply by $e^{\mp 2 \operatorname{Im} \kappa r} r^{-n+1+\delta}(\partial \bar{v} / \partial r)$ on both sides and take the real parts. Then the repeated use of integration by parts gives $(2 \cdot 4)$ if we note 


$$
\zeta_{ \pm}=e^{\mp i \kappa r} r^{-(n-1) / 2} D v \quad \text { and } \quad \theta_{ \pm}=\sum_{j=1}^{n} \frac{x_{j}}{r}\left[\zeta_{ \pm}\right]_{j}
$$

where $\left[\zeta_{ \pm}\right]_{j}$ is the $j$-th component of $\zeta_{ \pm}$.

Proposition 2. 1. Let $u$ be a $L^{2}$-solution of $(2 \cdot 1)$. Then there exists a constant $C_{2}>0$ such that for any $\kappa \in C-\mathbb{R}$

$$
|\kappa|^{2} \int_{\boldsymbol{R}^{n}}(1+r)^{-1-\delta}|u|^{2} d x \leq C_{2} \int_{\boldsymbol{R}^{n}}(1+r)^{1+\delta}|f|^{2} d x
$$

Proof. Multiply by $(1+\rho)^{-2 \delta} \rho^{-1+\delta}$ on both sides of $(2 \cdot 2)$ and integrate over $[0, \infty)$. Then we have

$$
\begin{aligned}
\frac{1}{2}|\kappa|^{2} & \int_{\boldsymbol{R}^{n}}(1+r)^{-2 \delta} r^{-1+\delta}|u|^{2} d x \\
& \leq \frac{1}{2} \int_{\boldsymbol{R}^{n}} r^{-1+\delta}\left|\theta_{ \pm}\right|^{2} d x+C(\delta) \int_{\boldsymbol{R}^{n}}|f \bar{i} \bar{u}| d x .
\end{aligned}
$$

On the other hand, noting that $n \neq 2,0<\delta \leq 1$ and $\left|\zeta_{ \pm}\right| \geq\left|\theta_{\Perp}\right|$, we have from $(2 \cdot 4)$

$$
\int_{\boldsymbol{R}^{n}} r^{-1+\delta}\left|\theta_{ \pm}\right|^{2} d x \leq\left(\frac{2}{\delta}\right)^{2} \int_{\boldsymbol{R}^{n}} r^{1+o}|f|^{2} d x
$$

Inequality $(2 \cdot 8)$ then follows if we note $(1+r)^{-1-\hat{o}} \leq(1+r)^{-2 \hat{\jmath}} r^{-1+\hat{o}}$.

\section{§3. Proof of Theorem $\mathbb{1}$}

(a) Let $f=\left\{f_{1}, f_{2}\right\} \in \mathcal{H}$. Then $u(t)=U(t, 0) f$ satisfies (1.4) and the initial condition $u(0)=f$. Since $\Lambda_{0}$ is skew-selfadjoint, we have from $(1 \cdot 4)$

$$
U_{0}(-t) U(t, 0) f=f-\int_{0}^{t} U_{0}(-\tau) V(\tau) U(\tau, 0) f d \tau
$$

and

$$
\|U(t, 0) f\|_{E}^{2}+2 \int_{0}^{t}\|\sqrt{V(\tau)} U(\tau, O) f\|_{E}^{2} d \tau=\|f\|_{E}^{2}
$$

We put

$$
A=\left(\begin{array}{cc}
0 & 0 \\
0 & a(x)
\end{array}\right), a(x)=\sqrt{C_{1}}(1+|x|)^{-(1+\delta) / 2} .
$$


Note that $A \geq \sqrt{V(t)}$. Then for any $g \in \mathcal{H}$

$$
\begin{aligned}
& \int_{s}^{t}\left|\left(U_{0}(-\tau) V(\tau) U(\tau, 0) f, g\right)_{E}\right| d \tau \\
& \quad \leq\left(\int_{s}^{t}\|\sqrt{V} \overline{(\tau)} U(\tau, 0) f\|_{E}{ }^{2} d \tau\right)^{1 / 2}\left(\int_{s}^{t}\left\|A U_{0}(\tau) g\right\|_{E}{ }^{2} d \tau\right)^{1 / 2},
\end{aligned}
$$

where $(,)_{E}$ denotes the inner product in $\mathcal{H}$. Thus, to see the existence of the strong limit of $(3 \cdot 1)$ as $t \rightarrow \infty$, it is sufficient to prove that there exists a constant $C_{3}>0$ such that

$$
\int_{0}^{\infty}\left\|A U_{0}(t) g\right\|_{E}^{2} d t \leq C_{3}\|g\|_{E}^{2} \quad \text { for any } \quad g \in \mathcal{H} .
$$

The following result is due to Kato [3].

Proposition 3. 1. There exists a $C_{3}>0$ satisfying (3.5) if the operator $A$ satisfies the condition

$$
\sup _{\kappa \in \boldsymbol{C}-\boldsymbol{R}}\left\|A\left(\Lambda_{0}-i \kappa I\right)^{-1} A\right\|_{E}<\infty .
$$

For $g=\left\{g_{1}, g_{2}\right\} \in \mathcal{H}$ put

$$
u=\left\{u_{1}, u_{2}\right\}=\left(\Lambda_{0}-i \kappa I\right)^{-1} A g .
$$

Then, as is easily seen, the second component $u_{2}$ satisfies equation $(2 \cdot 1)$ with $f=-i \kappa a(x) g_{2}$. Thus, by Proposition 2.1 we have

$$
\begin{aligned}
|\kappa|^{2} \int_{\mathbb{R}^{n}}(1+r)^{-1-\vartheta}\left|u_{2}\right|^{2} d x & \leq C_{2} \int_{\boldsymbol{R}^{n}}(1+r)^{1+\delta}\left|i \kappa a(x) g_{2}\right|^{2} d x \\
& \leq C_{1} C_{2}|\kappa|^{2} \int_{\mathbb{R}^{n}}\left|g_{2}\right|^{2} d x
\end{aligned}
$$

Since $A\left(\Lambda_{0}-i \kappa I\right)^{-1} A g=\left\{0, a(x) u_{2}\right\}$, it follows from (3.8) that

$$
\begin{gathered}
\left\|A\left(\Lambda_{0}-i \kappa I\right)^{-1} A g\right\|_{E}^{2}=\int_{\mathbb{R}^{n}}\left|a(x) u_{2}\right|^{2} d x \\
\leq C_{1}{ }^{2} C_{2} \int_{\boldsymbol{R}^{n}}\left|g_{2}\right|^{2} d x \leq C_{1} C_{2}\|g\|_{E}{ }^{2}
\end{gathered}
$$

This proves that $A$ satisfies condition (3.6). Hence, (3.5) holds and the wave operator $Z$ exists.

(b) To show the existence of $f \in \mathscr{H}$ such that $Z f \neq 0$, we assume 
contrary, i.e., for any $f \in \mathcal{H}\|U(t, 0) f\|_{E} \rightarrow 0$ as $t \rightarrow \infty$. Then we have from $(3 \cdot 2)$

$$
\|f\|_{E}^{2}=2 \int_{0}^{\infty}\|\sqrt{V} \overline{(t)} U(t, 0) f\|_{E}^{2} d t .
$$

Further, by $(3 \cdot 1)$ and $(3 \cdot 4)$

$$
\|f\|_{E}^{2} \leq\left(\int_{0}^{\infty}\|\sqrt{V(t)} U(t, 0) f\|_{E}^{2} d t\right)^{1 / 2}\left(\int_{0}^{\infty}\left\|A U_{0}(t) f\right\|_{E}^{2} d t\right)^{1 / 2} .
$$

Hence, it follows that

$$
\|f\|_{E}^{2} \leq \frac{1}{2} \int_{0}^{\infty}\left\|A U_{0}(t) f\right\|_{E}^{2} d t
$$

Put $f=U_{0}(s) g$, where $\|g\|_{E}=1$. Then by $(3 \cdot 12)$

$$
\left\|U_{0}(s) g\right\|_{E}^{2}=1 \leq \frac{1}{2} \int_{s}^{\infty}\left\|A U_{0}(t) g\right\|_{E}^{2} d t \rightarrow 0, \quad \text { as } \quad s \rightarrow \infty
$$

(cf., (3.5)). This is a contradiction and (b) is proved.

(c) It follows from (3.5) that in (3.4)

$$
\int_{s}^{t}\left\|A U_{0}(\tau) g\right\|_{E}^{2} d \tau \rightarrow 0 \quad \text { as } \quad s, t \rightarrow \infty
$$

On the other hand, we have from $(3 \cdot 2)$

$$
\int_{0}^{\infty}\|\sqrt{V} \overline{(t)} U(t, 0) f\|_{E}^{2} d t \leq \frac{1}{2}\|f\|_{E}^{2} \quad \text { for any } \quad f \in \mathscr{H} .
$$

Thus, $U(t, 0) * U_{0}(t) g$ converges in $\mathcal{H}$ as $t \rightarrow \infty$ and (c) is proved.

\section{$\S 4$. Proof of Theorem 2}

The assertions (a) and (b) for the operator $W$ can be proved by the same argument as in the proof of Theorem 1 if we note that the adjoint semigroup $U(t)^{*}$ has generator

$$
\Lambda^{*}=-\Lambda_{0}-V \quad \text { with domain } \mathscr{D}\left(\Lambda^{*}\right)=\mathscr{D}\left(\Lambda_{0}\right) .
$$

(c) and (d) are obvious from the definition of $W$ and $Z$.

\section{References}

[1] Iwasaki, N., On the principle of limiting amplitude, Publ. RIMS, Kyoto Univ. Ser A, 3 (1968), 373-392. 
[2] Kato, T., On linear differential equations in a Banach space, Comm. Pure Appl. Math., 9 (1956), 479-486.

[3] Kato, T., Wave operators and similarity for some non-selfadjoint operators, Math. Ann., 162 (1966), 255-279.

[4] Lax, P. D. and Phillips, R. S., Scattering theory for dissipative hyperbolic systems, J. Functional Analysis, 14 (1973), 172-235.

[5] Mizohata, S. and Mochizuki, K., On the principle of limiting amplitude for dissipative wave equations, J. Math. Kyoto Univ., 6 (1966), 109-127.

[6] Mochizuki, K., Spectral and scattering theory for second order elliptic differential operators in an exterior domain, Lecture Notes Univ. Utah, Winter and Spring 1972.

[7] Mochizuki, K., Decay and asymptotics for wave equations with dissipative term, Lecture Notes in Physics 39 (International Symposium on Mathematical Problems in Theoretical Physics), Springer-Verlag, Berlin·Heidelberg $\cdot$ New York 1975, 486-490.

Added in Proof. Recently, Mr. A. Matsumura (Dept. Appl. Math. Phys., Fac. Engi., Kyoto U.) obtained the following result: If $b(x, t)$ in $(1 \cdot 1)$ satisfies $t \geq 0$

$$
b_{t}(x, t) \leq 0 \text { and } \min _{|x| \leq R+t} b(x, t) \geq \frac{1}{K+\varepsilon t},
$$

where $R, K, \varepsilon$ are positive constants, and if the initial data $f=\left\{f_{1}, f_{2}\right\}$ has support contained in $\{x ;|x| \leq R\}$, then the total energy of solution of $(1 \cdot 1)$ decays like

$$
\|U(t, 0) f\|_{E}=O\left(t^{-1 /(2+3 \varepsilon)}\right) \text { as } t \rightarrow \infty .
$$

By this result we can say that our assumption (A1) is settled in a sense. 